\title{
Susanne Rau, Räume: Konzepte, Wahrnehmungen,
}

Nutzungen

Frankfurt/M., New York : Campus (Historische Einführungen, 14), 2013, 237 p., $16,90 €$

Falk Bretschneider

\section{(2) OpenEdition}

\section{Journals}

Édition électronique

URL : http://journals.openedition.org/ifha/8145

DOI : 10.4000/ifha.8145

ISSN : 2198-8943

\section{Éditeur}

IFRA - Institut franco-allemand (sciences historiques et sociales)

\section{Référence électronique}

Falk Bretschneider, "Susanne Rau, Räume: Konzepte, Wahrnehmungen, Nutzungen », Revue de l'IFHA [En ligne], Date de recension, mis en ligne le 14 avril 2015, consulté le 22 septembre 2020. URL : http:// journals.openedition.org/ifha/8145; DOI : https://doi.org/10.4000/ifha.8145

Ce document a été généré automatiquement le 22 septembre 2020

(CIFHA 


\section{Susanne Rau, Räume: Konzepte, Wahrnehmungen, Nutzungen}

Frankfurt/M., New York : Campus (Historische Einführungen, 14), 2013, 237 p., $16,90 €$

Falk Bretschneider

L'importance et la place d'une problématique au sein d'une discipline se mesurent aussi à l'aune des manuels qui lui sont consacrés. Ces ouvrages constituent souvent une certaine cristallisation des connaissances qui sont considérées désormais, du moins en partie, comme acquises; mais ils peuvent parfois aussi afficher un caractère plus programmatique et donc esquisser les contours d'un champ de recherche qu'il s'agit encore d'explorer. Consacré au domaine des recherches historiques sur l'espace, le présent ouvrage fait indéniablement partie du deuxième groupe, en reflétant par làmême la situation particulière de cet objet dans l'historiographie allemande. En France, la plupart des historiens ont accueilli avec étonnement, voire avec un certain amusement, les débats sur les études spatiales apparus depuis une dizaine d'années à l'échelle internationale. Forte d'une tradition disciplinaire particulière (les liens anciens et profonds entre histoire et géographie), l'historiographie française n'avait certainement pas besoin d'un «tournant spatial» pour s'intéresser aux dimensions spatiales dans l'histoire. En Allemagne, la situation est toute autre : ce même intérêt y ressemblait parfois, ces dernières années, à la voracité d'un affamé suivie d'un rassasiement inévitable, mais peu satisfaisant : après de longues années de scepticisme généralisé ou même d'ignorance envers l'espace, notion profondément contaminée par l'idéologie national-socialiste, l'attention qu'on se mit à lui porter autour de l'an 2000, dans le sillage d'une réorientation de la discipline vers les problèmes d'un monde globalisé, fut effectivement nouvelle, donnant lieu à une véritable cascade de colloques, de numéros de revues ou d'ouvrages collectifs consacrés à la thématique. Toutefois, cet engouement fut de courte durée, laissant derrière lui une discipline dont l'intérêt pour l'espace n'allait souvent guère au-delà d'une remise au goût du jour d'objets et de méthodes anciens, et au sein de laquelle les études proposant un regard véritablement 
nouveau sur l'histoire de l'espace et sur l'espace dans l'histoire sont toujours aussi peu monnaie courante.

Tous ces aspects sont également évoqués dans ce volume de la collection " introductions historiques ", qui se donne pour but de proposer à la jeune recherche en histoire une première orientation dans cette jungle des débats qui, depuis plus d'un siècle, sont menés autour de l'espace dans toutes les disciplines des sciences humaines et sociales. Ceci explique aussi le titre de l'ouvrage: "espaces" (et non pas, par exemple, "histoire de l'espace ») car son objectif dépasse celui d'un simple guide destiné à circonscrire les fondements d'une nouvelle sous-discipline historique. À l'instar d'autre champs scientifiques, celui des recherches sur le genre par exemple, il s'agit donc plutôt d'attirer l'attention de l'ensemble des historiens sur une problématique appartenant en fait, du moins dans les intentions de l'auteure, à l'histoire générale. Comme c'est souvent le cas en Allemagne, une telle approche commence par une démarche théorique, meilleur moyen d'assurer à un nouvel objet la dignité d'une catégorie universelle. L'auteure, occupant à l'université d'Erfurt une chaire consacrée à l'histoire et aux cultures des espaces à l'époque moderne, propose par conséquent dans les deux premières parties de l'ouvrage d'appréhender la thématique par le biais des concepts et des notions. Susanne Rau y évoque d'abord les différentes idées que se sont faites de l'espace les philosophes et autres penseurs, allant de l'Antiquité (Platon, Aristote) jusqu'aux conceptions relationnelles de Leibniz ou d'Einstein, pour ensuite retracer « la carrière » plus particulièrement « allemande » du concept, en insistant sur le destin fatal, car susceptible de détournements politiques, d'une démarche marquée par l'idée d'un déterminisme spatial tel qu'il apparait dans les travaux de Carl Ritter, Friedrich Ratzel ou Carl Schmitt. À juste titre, les études de Paul Vidal de La Blache, Lucien Febvre, Fernand Braudel et surtout Henri Lefebvre incarnent une alternative théorique et méthodologique à cette impasse, en menant peu à peu à une conception de l'espace qui n'y voit plus quelque chose de donné, extérieur à l'action humaine, mais le produit de la vie en société. Dans cette même perspective, la deuxième partie de l'ouvrage est consacrée aux approches d'autres sciences sociales susceptibles de fournir aux historiens les bases d'un travail qui ne saurait être qu'interdisciplinaire. Composée d'exemples issus principalement de la géographie, de l'anthropologie culturelle et des études postcoloniales ainsi que de la sociologie, cette liste d'apports possibles se distingue par sa grande richesse et sa variété (il y manque peut-être le concept des «non-lieux » de Marc Augé) ; elle permet ainsi au lecteur de se plonger lui-même dans l'univers des diverses positions théoriques et d'y tracer son propre chemin de lecture. Ceci vaut également pour les propositions plus particulièrement historiques (Reinhart Koselleck et Karl Schlögel en Allemagne, Michel de Certeau, Jean-Claude Perrot et Bernard Lepetit en France) qui clôturent cette partie.

En avançant dans la lecture, on comprend cependant très vite que Susanne Rau n'est guère satisfaite de la manière dont les historiens traitent pour l'instant de l'espace $(\mathrm{y}$ compris en France). C'est pourquoi elle propose elle-même, dans la troisième et la plus importante partie de l'ouvrage, un schéma analytique qui se veut un véritable mode d'emploi opérationnalisant les résultats des discussions conceptuelles menées dans les chapitres précédents. Le lecteur n'y trouvera pas seulement une réflexion sur les sources disponibles pour une analyse historique de l'espace, mais surtout une démarche détaillée (dont il est impossible ici de résumer l'ensemble des éléments) procédant en quatre étapes consacrées respectivement à l'étude (1) de la constitution de l'espace, (2) de ses dynamiques et transformations, (3) de ses perceptions et 
représentations subjectives ainsi que (4) de ses usages et appropriations par les acteurs sociaux. Pour donner des contours plus concrets à cette proposition, l'auteure l'accompagne de deux petites études de cas, l'une s'intéressant aux configurations spatiales du monde urbain (un objet sur lequel elle a travaillé elle-même), l'autre aux interactions créatrices d'espaces du commerce. Au total, Susanne Rau propose donc une vision convaincante de l'espace conçu comme une construction sociale et culturelle et donne en même temps au lecteur les moyens concrets de s'aventurer lui-même dans une analyse réflexive des dimensions spatiales de la société. Comme chaque volume de la collection, l'ouvrage est en outre complété par une bibliographie sélective, des sources supplémentaires et d'autres matériaux (par exemple un glossaire) mis à la disposition du lecteur sur internet à l'adresse http://www.historischeeinfuehrungen.de.

Évidemment, l'ouvrage de Susanne Rau a d'abord été écrit pour s'adresser à un public allemand. Le lecteur français pourrait ainsi être un peu irrité par le caractère souvent très théorique de la démonstration et par une démarche conceptuelle dont on peut avoir l'impression qu'elle s'intéresse parfois davantage à un travail notionnel poussé qu'au potentiel d'interprétation qu'offrent les sources elles-mêmes. Il pourrait également s'étonner de voir le travail cartographique, élément central de la méthode pour beaucoup d'historiens français, être relégué à un seul paragraphe (p. 194). Mais la démarche proposée par Susanne Rau ne s'est pas seulement nourrie de nombreuses études françaises, elle représente également une autre approche de l'espace. Au-delà de l'aperçu fort utile qu'il propose sur les enjeux afférents à l'espace dans l'ensemble des sciences sociales, son livre témoigne aussi d'une manière différente, s'inscrivant dans une autre tradition historiographique, d'analyser l'espace et son histoire - et en cela, il devrait intéresser également un public français.

INDEX

Index chronologique : Ouvrages transpériodiques

Thèmes : Manuels/Ouvrages généraux/Outils de travail

\section{AUTEUR}

FALK BRETSCHNEIDER

EHESS 